\title{
PAPR Reduction in OFDM System using Wavelet SLM and PTS Technique
}

\author{
Ajit Singh Rathor \\ UTU, Uttrakhand \\ ECE Department \\ J.B.I.T. Dehradun
}

\author{
Mukesh Pathela \\ UTU, Uttrakhand \\ H.O.D. ECE Department \\ SCE, Dehradun
}

\author{
Sultan Singh Rana \\ UTU, Uttrakhand \\ ECE Department \\ J.B.I.T. Dehradun
}

\begin{abstract}
Recently researchers have developed many techniques to solve high PAPR problem. These techniques have some merits and demerits. The concentration of this research work is especially upon the Selected Mapping (SLM) and Partial Transmit Sequence (PTS) techniques using Wavelet transforms (IDWT and DWT) instead of conventional Fast Fourier Transforms. The Fourier transform is a powerful tool to analyze the components of stationary signals for example this transform is used to process the signals containing the combination of sine and cosine signals. But the Fourier transform is less useful to analyze the components of non stationary signals. In non stationary signals there is a change in the properties of the signal and these signals are analyzes in real time. The wavelet transform is used to analyze the components of non stationary signals. Using wavelets the filters for stationary and non stationary signals can also be constructed. The mathematics of wavelets is much large in comparison to Fourier transform and this can be said that mathematics of wavelets encompasses the Fourier transform. The size of wavelet theory is matched to the application area. The main difference between these two transforms is the wavelet transform can be localized in both frequency and time domain whereas the Fourier transform can only be localized in frequency [11].
\end{abstract}

\section{Keywords}

Orthogonal Frequency Division Multiplexing Technique (OFDM), Selective Level Mapping (SLM), Partial Transmit Sequence (PTS), Peak to Average Power Ratio (PAPR), Inverse Discrete Wavelet Transform (IDWT) and Discrete Wavelet Transform (DWT).

\section{INTRODUCTION}

With the advance of communication technology the demand of high data rate services as multimedia, voice and data over wired and wireless links is increasing. New modulation schemes are required for transmitting the large amount of data. Over the last few years OFDM modulation scheme has performed the better result. OFDM [1] offers a very high data rate transmission in wireless communication system. OFDM is frequency division multiplexing scheme used as a digital multicarrier modulation method. Each subcarrier is modulated by various modulation schemes at low symbol rate in order to maintain total data rate equal to single carrier modulation scheme within the same bandwidth. These days OFDM technique is being considered as the important technique for the fourth generation (4G) mobile communication systems. OFDM has been used for Digital Audio Broadcasting (DAB) and Digital Video Broadcasting (DVB) in Europe. OFDM [1] has many properties better than the existing single carrier communication system and the prime property is the immunity to frequency selective fading. Single carrier communication systems can increase their data rate by making the symbol time short but by doing this the occupied bandwidth gets increased. Wideband channels also suffer from frequency selective fading and due to this these channels require complex equalizers at receiver side in order to recover original signal. In OFDM this problem is solved by dividing the wideband channel into a series of narrowband channels and these all narrowband channels experience flat fading. Therefore only one tap equalizer is required at receiver side in order to recover original signal, so by this method the complexity of receiver is reduced to a great extent. OFDM system uses the advance silicon and Digital Signal Processing in order to use efficient Fourier transforms at the transmitter and receiver to perform modulation and demodulation respectively. The efficiency of bandwidth is very good in OFDM system because the subcarriers are orthogonal and overlap to each other. These orthogonal and overlap subcarriers are easily decoded at receiver. OFDM system also does not suffer from the basic transmission problems as large attenuation, noise, interference, time variance and nonlinearities.

Besides of these advantages OFDM system suffers from a big disadvantage $[1,2]$ that this system has a large value of Peak to Average Power Ratio (PAPR) or large Envelope Fluctuation. This large PAPR occurs due to superposition of $\mathrm{N}$ independent equally spaced subcarriers at the output of Inverse Fast Fourier Transform (IFFT) in the transmitter section of the system. Due to this high value of PAPR the signal peaks move into the nonlinear region of RF power amplifier which creates the distortion in the signals [4]. A large PAPR causes complexity at the output of Inverse Fast Fourier Transform (IFFT) and Digital to Analog Converter (DAC).

But the most serious problem created by high value of PAPR is the reduction in the efficiency of High Power Amplifier (HPA) which must be avoided. Also a high value of PAPR can saturate the high power amplifier due to which out of band radiations are produced. These radiations affect adjacent channels and degrade the Bit Error Rate (BER) at the receiver. There are many techniques to minimize this high PAPR problem. The Clipping and Filtering is the very basic technique to reduce the PAPR. Other techniques like Coding, Companding, Tone Reservation (TR), Tone Injection (TI), Selected Mapping (SLM) [3, 4] and Partial Transmit Sequence (PTS) are also being used to reduce the high value of PAPR. The PTS technique provides better PAPR reduction performance than SLM but transmitter and receiver complexity is very high in the PTS technique also this needs [4] transmission of side information about selected sequence to the receiver in order to cancel the rotation of OFDM sub carriers. The concentration of this paper is especially upon the 
Selected Mapping (SLM) and Partial Transmit Sequence (PTS) techniques. Wavelet transforms instead of conventional Fast Fourier Transforms are being used in these techniques. Out of these two techniques the Selective Level Mapping (SLM) technique has low value of PAPR.

\section{PEAK TO AVERAGE POWER RATIO IN OFDM SYSTEM}

The PAPR is defined as the ratio of maximum power and average power for the envelope of a baseband complex signal $s(t)$.

$$
P A P R=10 \log 10 \frac{\max _{0 \leq t \leq T} \mathrm{I} x(t) \mathrm{I}^{2}}{P_{a v}}
$$

Where $P_{a v}$ is the average power of the signals in time domain, and $x(t)^{2}$ is the maximum peak of the signal.

The input to the amplifier in OFDM system in analog signal, from the IFFT the time domain samples of output can be written as under.

$$
x[n]=\frac{1}{\sqrt{N}} \sum_{i=0}^{N-1} X(i) e^{(j 2 \pi i n) / N}, 0 \leq \mathrm{n} \leq \mathrm{N}-1
$$

If the number of subcarriers is larger than $x[n]$ are zero mean Gaussian random variables. The OFDM signal is Rayleigh distributed for with variance $\sigma_{n}^{2}$ for $\mathrm{x}[\mathrm{n}]$ complex Gaussian and phase of the signal is uniform. The peak value of the signal having Rayleigh distribution will crossover any values with non zero probability.

\subsection{Probability Distribution Function of PAPR}

The cumulative distribution function (CDF) is a parameter used to measure the efficiency of any PAPR reduction technique [2]. In most cases the Complementary CDF or CCDF is used in place of CDF which finds the probability that PAPR of a certain data block has exceeded a given threshold limit [9]. The real and imaginary parts of time domain signal have a zero mean and variance of 0.5 and also follow the Gaussian distribution, this happens when central limit thermo is implemented for multi carrier signal with large number of subcarriers. The Rayleigh distribution is being followed for the amplitude of a multi carrier signal and central chi square distribution is followed for the power distribution of the system with two degree of freedom.

The CDF for the amplitude of a signal sample is

$$
\mathrm{F}(\mathrm{z})=1-\exp (\mathrm{z})
$$

The CCDF of PAPR of data block is used to compare the output of various reduction techniques is given by

$$
\begin{aligned}
\mathrm{P}(\mathrm{PAPR}>\mathrm{z}) & =1-\mathrm{P}(\mathrm{PAPR} \leq \mathrm{z}) \\
& =1-\mathrm{F}(\mathrm{z})^{\mathrm{N}} \\
& =1-(1-\exp (-\mathrm{z}))^{\mathrm{N}}
\end{aligned}
$$

\section{PAPR REDUCTION TECHNIQUES}

To reduce the High value of PAPR in OFDM systems there are many techniques available and these techniques vary according to the need of the system and dependent on various factors [8]. These factors are as PAPR reduction capacity, increase in power of transmit signal, loss in data rate, complexity of computation and increase in BER at receiver. These techniques can be classified in the three categories.

\subsection{Signal Distortion}

Clipping is the simple technique in OFDM system used to reduce the high value of PAPR. In this technique the higher peak of the signal coming in outside of allowed region is removed or clipped $[7,10]$

\subsection{Coding Technique}

The coding techniques are based on the principle that the code which reduces the PAPR is selected because all codes do not give high PAPR. This does not cause any distortion and out of band radiation. But due to this scheme the bandwidth efficiency is degraded as this reduces the code rate. There are many coding schemes to reduce the PAPR as Linear block coding, Turbo coding and Golay codes.

\subsection{Signal Scrambling Techniques}

In these techniques the scrambling of input sequence is done by some scrambling sequence for each OFDM symbol [6]. After this the signal having lowest PAPR is selected and transmitted. These techniques do not give the guarantee to offer PAPR below some level but decreases the probability of occurrence of high value PAPR. There are mainly two scrambling techniques the Selected Mapping (SLM) and Partial Transmit Sequence (PTS).

\section{WAVELET TRASFORMS}

Wavelet transform was proposed as an alternative of the FFT systems. This wavelet transform was introduced by Jean Morlet in 1982 and this was a new mathematical tool for seismic wave analysis [11]. The wavelet transform decomposes the desired signal into the set of basis waveforms which are called wavelets. A wavelet can be defined a small wave which concentrates its energy in the time and gives a tool for the analysis of transient or time varying phenomena [11]. The wavelets are derived from a multistage tree structured Haar and Daubechies orthonormal QMF bank. In the wavelet transform the multi resolution analysis is performed in which an input signal is decomposed into different frequency components and after this study of each component with the resolution matched to its scales is done. This is the same process which is done in Fourier transform but the difference is that here the basis functions are not the sine or cosine waves. Due to finite duration the wavelet has both time and frequency localization and can combat the effect of ISI efficiently without using CP. At higher frequencies the wavelets offer good time resolution and poor frequency resolution and at lower frequencies wavelets offer good frequency resolution and poor time resolution. The wavelet transforms are of two types one is continuous wavelet transform (CWT) and other is discrete wavelet transform (DWT). In this research work the IDWT and DWT are being used to reduce the PAPR.

\section{PROPOSED WAVELET SLM TECHNIQUE}

The first proposed scrambling technique is known as Selected Mapping (SLM) technique and this has been used to get the reduction in PAPR. Here a new technique is being introduced which uses Wavelet Transforms instead of Fast Fourier Transforms in the SLM technique to minimize the PAPR and simulation is being done on Mat lab platform. The PAPR has been reduced to a great extent by using this new technique. Figure 1 shows the block diagram of SLM transmitter using Inverse Discrete Wavelet Transform (IDWT) instead of Inverse Fast Fourier Transform (IFFT) [2,6]. The data is first 
split into blocks then the data, $\mathrm{X}\left(\mathrm{X}_{0}, \mathrm{X}_{1}, \mathrm{X}_{2} \ldots \ldots . \mathrm{X}_{\mathrm{N}-1}\right)^{\mathrm{T}}$ is multiplied by the individual phase sequence (or phase vector) which is indicated like $\mathrm{P}^{\mathrm{u}}=\left(\mathrm{P}_{0}{ }^{\mathrm{u}}, \mathrm{P}_{1}{ }^{\mathrm{u}}, \mathrm{P}_{2}{ }^{\mathrm{u}} \ldots \ldots \ldots \mathrm{P}_{\mathrm{N}-1}{ }^{\mathrm{u}}\right)^{\mathrm{T}}$ where $\mathrm{u}$ is the number of phase sequence or phase vector $(\mathrm{u}=0$, $1, \ldots \ldots \ldots . . . \mathrm{U}-1)$. After the multiplication process the IDWT operation is applied to each phase sequence in order to covert the signal from frequency domain to time domain. The multiplication process gives the data block OFDM system and this has the different time domain signals of length U. Further this data block of OFDM system has the different PAPR values which is indicated as $\mathrm{Xu}=\left(\mathrm{X}_{0}{ }^{\mathrm{u}}+\mathrm{X}_{1}{ }^{\mathrm{u}}+\mathrm{X}_{2}{ }^{\mathrm{u}}\right.$ $\left.+\ldots \ldots \ldots+X_{N-1}{ }^{u}\right)^{T}$. In the last step the PAPR of different independent data blocks is compared and the data block having lowest PAPR is selected for the transmission

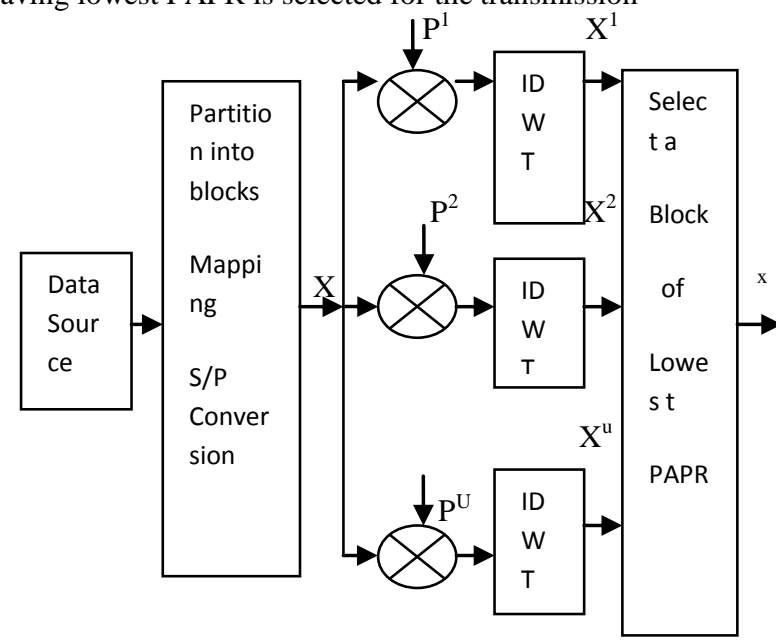

Figure 1: The block diagram of SLM transmitter using wavelet transform

The following equation shows the suitable candidate which is being selected for the transmission purpose.

$$
\mathrm{X}^{\wedge}=\operatorname{argmin}_{0 \leq \mathrm{u} \leq \mathrm{U}}\left[\operatorname{PAPR}\left(\mathrm{X}^{\mathrm{u}}\right)\right]
$$

In this technique in order to generate the alternative OFDM symbols the independent phase sequence should be generated. The $\mathrm{k}^{\text {th }}$ value of $\mathrm{u}^{\text {th }}$ phase sequence can be written as.

$$
\mathrm{P}^{\mathrm{u}}(\mathrm{k})=\mathrm{e}^{\mathrm{j} \phi(\mathrm{u})(\mathrm{k})}
$$

Here $\phi(\mathrm{k})$ is the random phase value. $\mathrm{X}^{\mathrm{u}}$ is phase rotated version of $X$, the two phase sequence or phase vector are said to be dependent if any joint cumulate between them is nonzero. So the condition of mutual independent between two phase vectors can be written as.

$$
E\left[\mathrm{e}^{\mathrm{j} \phi}\right]=0
$$

This is satisfied if $\phi$ is distributed uniformly in [0 2 [ 2 ]

The OFDM signal which is selected at transmitter will have to be detected at receiver and to do this receiver should have the knowledge of multiplied phase sequence used to generate that particular OFDM symbol at transmitter side. So in order to accomplish this task perfectly some side information (SI) is being transmitted with the selected OFDM signal. This side information (SI) index is transmitted as a set of $\left[\log _{2} \mathrm{U}\right]$ bits.

\section{PROPOSED WAVELET PTS TECHNIQUE}

The second proposed scrambling technique is known as Partial Transmit Sequence (PTS) used to reduce PAPR in OFDM system..

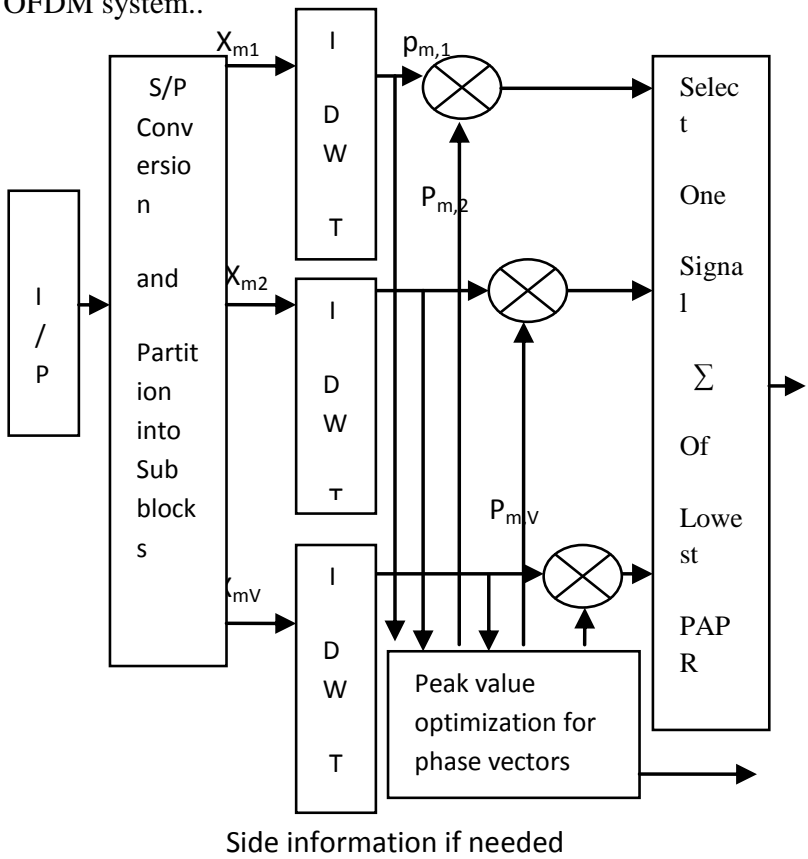

Figure 2: The block diagram of PTS transmitter using wavelet transform

This method is based on the two main steps. In the first step the original OFDM signal is divided into number of sub blocks and in the second step each sub block is multiplied by a phase factor and then the phase rotated sub blocks are added together in order to generate the number of candidate signals and among these candidates the lowest PAPR signal is selected for the transmission. Figure 2 shows this scheme [6, 10 , and 12]. The working principle of this scheme is quite similar to the conventional PTS scheme the only difference is that this proposed scheme uses the Wavelet Transforms (IDWT and DWT) instead of Fast Fourier Transforms (IFFT and FFT)

The original OFDM signal is shown in the below equation (8)

$$
\mathrm{x}_{\mathrm{m},=}=\frac{1}{\sqrt{N}} \sum_{k=0}^{N-1} X m e^{(j 2 \pi m k) / N} \quad 0 \leq \mathrm{n} \leq \mathrm{N}-1 \ldots .
$$

The transmitted signal will be divided into several sub blocks, $\mathrm{X}_{\mathrm{m}, \mathrm{k}}$, of length $\mathrm{N} / \mathrm{V}$. Where $\mathrm{N}$ is the number of subcarriers and $\mathrm{V}$ is the number of sub blocks. Now all the positions of subcarriers are set to zero which are occupied in another block [10], this gives the $X_{m}=\sum_{k=1}^{V} X_{m k}$. After this a constant phase rotation of $\mathrm{p}_{\mathrm{mk}}=\mathrm{e}^{\mathrm{j} \text { कmk }}$ is applied to each sub block where $\phi_{\mathrm{mk}} \in[0,2 \Pi], 1<\mathrm{k}<\mathrm{V}$. The phase rotation is not applied on first block which is constant. This resultant signal contains the same information as $\mathrm{X}_{\mathrm{m}}$ but only the phase rotation is being added and this phase rotation must be known to the receiver. An IDWT operation is performed on each sub block and after this these all are added together to create a possible symbol for transmission. This process will be repeated again with the different phase rotation $\mathrm{p}_{\mathrm{mk}}$ in order to generate another alternative transmit signals. Here $\mathrm{x}_{\mathrm{mk}}$ is the partial transmit sequence. 


\section{SIMULATION AND RESULTS}

In this section the performance of the proposed techniques namely SLM and PTS using wavelet transforms (IDWT and DWT) will be evaluated. The simulation results show that the PAPR has been reduced in a great manner by wavelet SLM and PTS techniques.

\subsection{PAPR Reduction with BPSK modulation}

Figure 3 shows the PAPR reduction using PTS technique at receiver side of an OFDM system. Here the DWT has been used at the receiver side of the system. Figure 4 shows the performance of two PAPR reduction techniques namely SLM and PTS by making the comparison with original signal using BPSK modulation. BPSK modulator object modulates using the binary phase shift keying method and the output is baseband representation of the modulated signal. The BPSK demodulator object demodulates the signal that was modulated using the binary phase shift keying method and the input is baseband representation of the modulated signal. The number of subcarriers $(\mathrm{N}=256)$ and oversampling factor $(\mathrm{L}=4)$ are same for both of the techniques. Both of the techniques can reduce the PAPR of an OFDM system in a significant manner. This is cleared from the figure that the PTS technique performs better than the SLM technique.

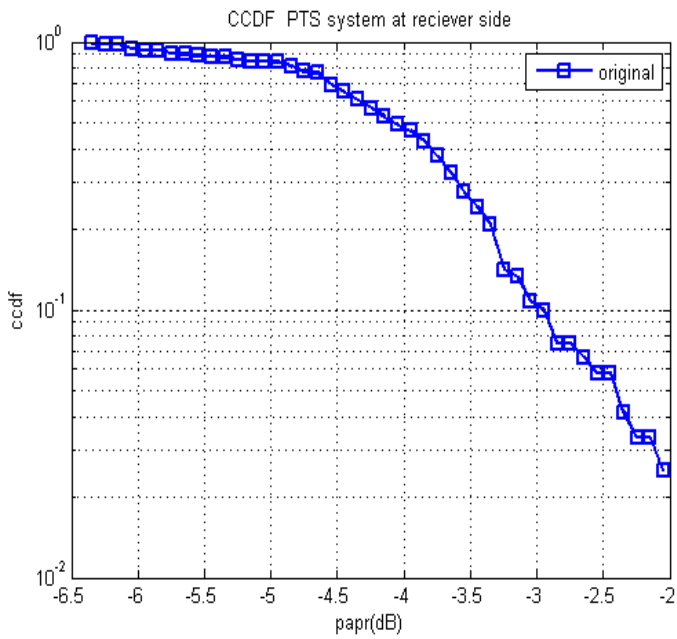

Figure 3: PAPR reduction in OFDM system using PTS technique with BPSK

Figure 5 shows the results of probability of bit error (BER) performance for both the case of simulation and theoretical with BPSK modulation. This comparison of BER between simulation and theoretical was carried out to make sure that the results of simulink model are correct. This is evident that simulation curve is close to the theoretical curve which is desired.

\subsection{PAPR Reduction with QPSK modulation}

This section describes the PAPR reduction by using QPSK (Quadrature Phase Shift Key) modulation technique. Figure 6 shows the PAPR reduction using PTS technique at receiver side of an OFDM system and the QPSK modulator and demodulator has been used in this process.

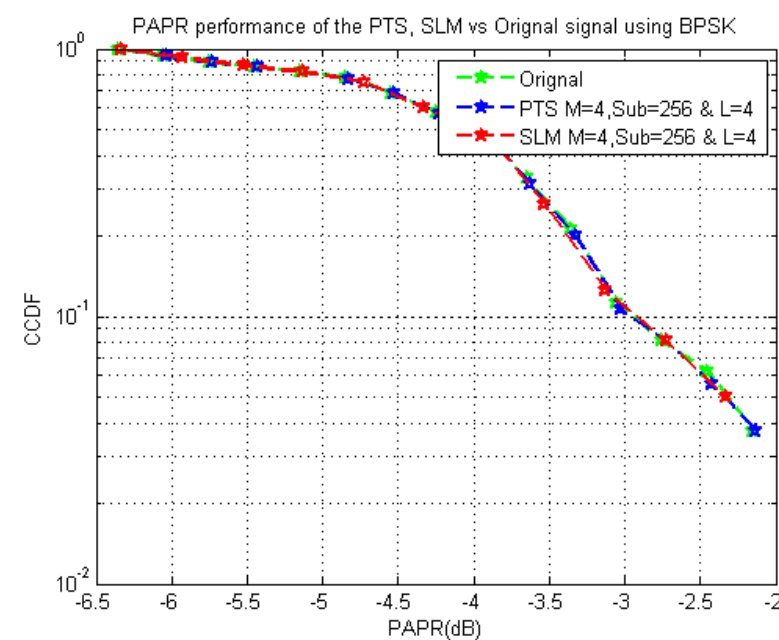

Figure 4: Comparison of PTS and SLM techniques with BPSK modulation

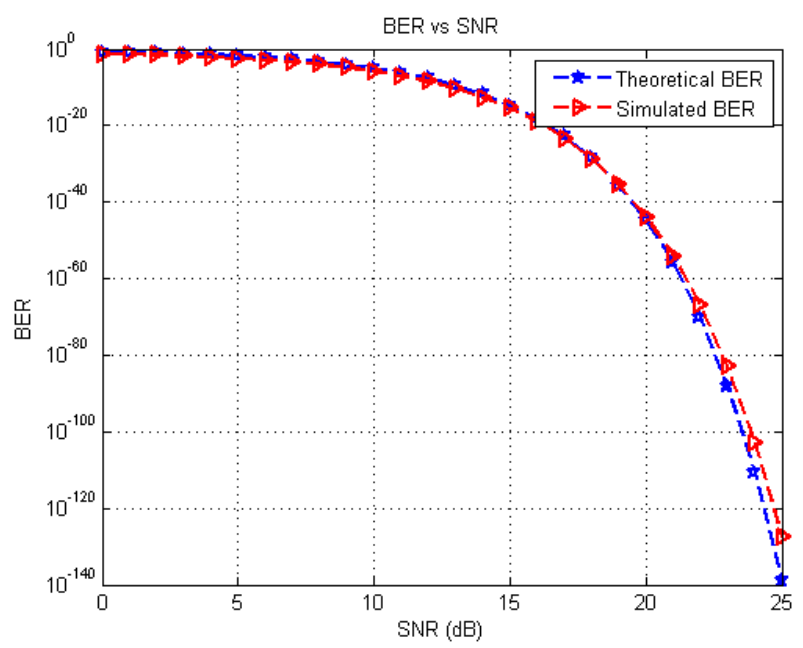

Figure 5: Simulated BER versus theoretical BER with BPSK Modulation

QPSK modulator object modulates using the quaternary phase shift keying method and the output is baseband representation of the modulated signal. The QPSK demodulator object demodulates the signal that was modulated using the quaternary phase shift keying method and the input is baseband representation of the modulated signal. From figure 6 this is clear that PAPR has been reduced to a great extent. Figure 7 shows the performance of two PAPR reduction techniques namely SLM and PTS by making the comparison with original signal using QPSK modulation method. 


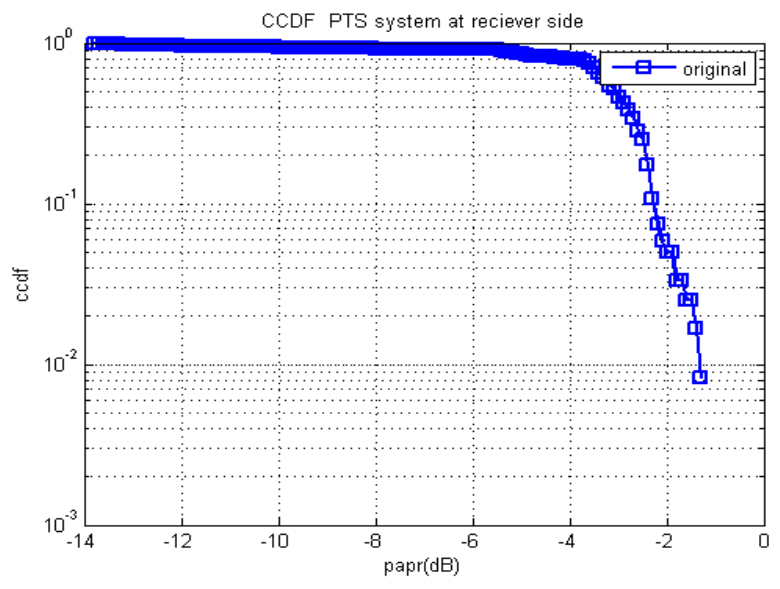

Figure 6: PAPR reduction in OFDM system using PTS technique with QPSK

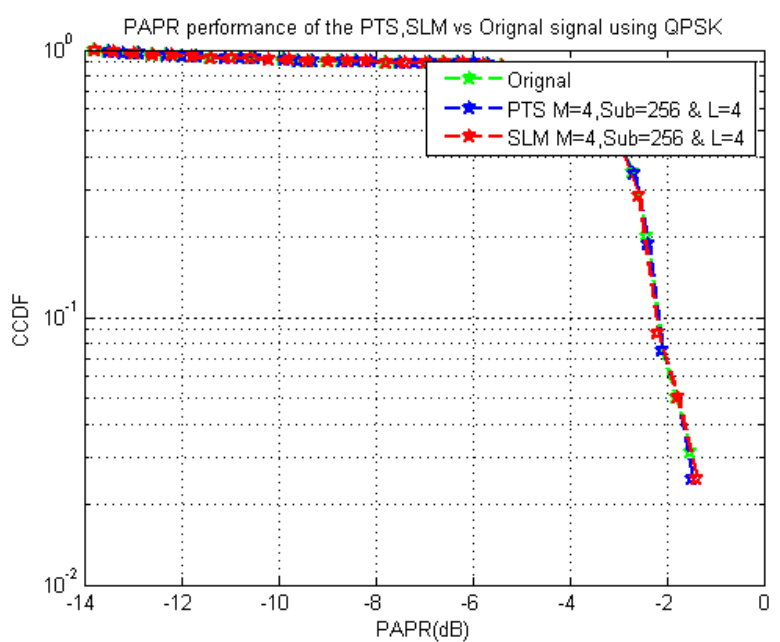

Figure 7: Comparison of PTS and SLM techniques with QPSK modulation

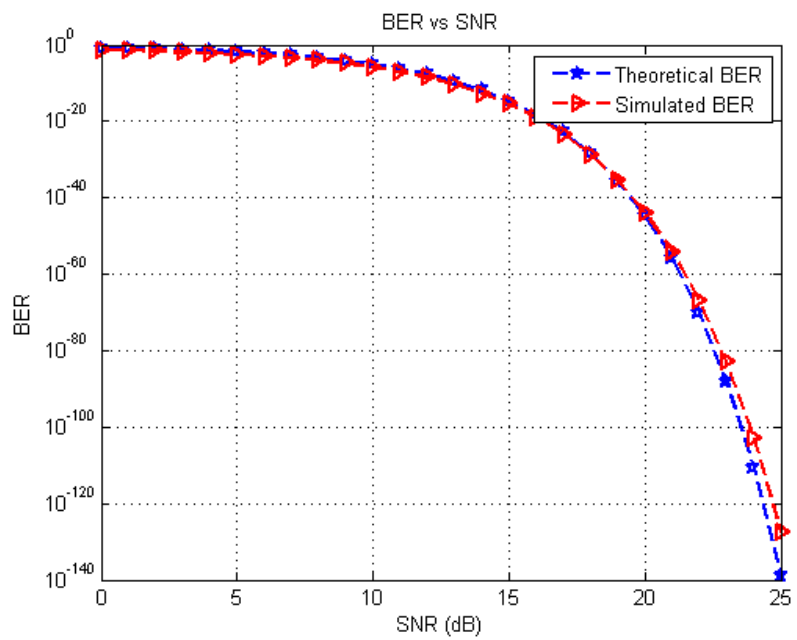

Figure 8: Simulated BER versus theoretical BER with QPSK modulation
Figure 8 shows the results of probability of bit error (BER) performance for both the case of simulation and theoretical with QPSK modulation. The simulation curve is close to the theoretical curve which is desired.

\subsection{Comparison of PAPR reduction by BPSK, QPSK and OQPSK Modulation}

In this section the simulation results of PTS technique at receiver side of an OFDM system using different modulation methods namely BPSK, QPSK, OQPSK are being compared. The figure 9 shows the simulation results and comparison of these modulation formats is also being shown in this figure. And this is clear from the figure that the QPSK (Quadrature Phase Shift Key) modulation technique gives the best result for PAPR reduction in comparison of BPSK and OQPSK modulation method. So in order to achieve the PAPR reduction efficiently the QPSK modulation method should be preferred in OFDM systems.

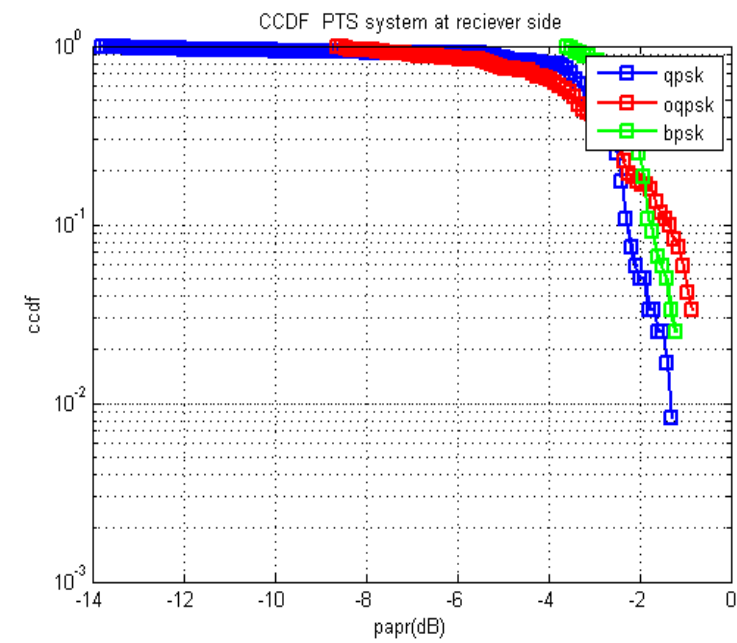

Figure9: Comparison of BPSK, QPSK, OQPSK modulation methods

\section{CONCLUSION}

OFDM system has been discussed in this paper work and this work indicates that OFDM is a popular communication system due its many advantages like its ability to convert the frequency selective fading channels to flat fading channels and the robustness to the ICI and ISI. SLM and PTS techniques using wavelets transforms (IDWT and DWT) were in the prime focus of this paper. Both techniques were explained in this paper with different modulation methods. The simulation results of this paper show that both of the techniques can reduce PAPR efficiently but PTS with QPSK modulation method is more effective in PAPR reduction.

\section{REFERENCES}

[1] Bahubali K. Shiragpur, Yday Wali, Sandeep Bidwai "Novel Technique to Reduce PAPR in OFDM system Using Threshold SLM", $20133^{\text {rd }}$ IEEE International Advance Computing Conference (IACC).

[2] B.Venkatesu and Karunaiah.B. "New Approach to Peak to Average Power Ratio Techniques for OFDM signals" International Journal of Artificial Intelligence and Mechatronics (IJAIM), Volume 1, Issue 4, ISSN 23205121,2013 
[3] E.Kalaiselvan, P.Elavarsan and G. Nagarajan "PAPR Reduction of OFDM Signals using Pseudo Random PTS without Side Information", IEEE, International conference on Communication and Signal Processing, April 3-5, 2013, India.

[4] Er. Ankita and Ananad Nayyar "Review of Various PTS Techniques of PAPR Reduction in MIMO OFDM", International Journal of Innovative Technology and Exploring Engineering (IJITEE), ISSN: 2278-3075, Volume -2, Issue-4, and March 2013.

[5] Ibrahim Mohammad Hussain "Low Complexity Partial SLM Technique for PAPR Reductin in OFDM Transmitters", International Journal on Electrical Engineering and Informatics, Volume5, November1, March 2013.

[6] Maan Singh and Vijay Kumar "Signal Scrambling Techniques for PAPR Reduction in OFDM systems", International Journal of Engineering and Computer Science, ISSN: 2319-7242, Volume -2, Issue-1, and Jan 2013.

[7] Navneet Kaur and Lavish Kansal "Peak to Average Power Ratio Reduction of OFDM Signals by Combining Clipping with Walsh Hadamard Transform", International Journal of Wireless and Mobile Networks
(IJWMN), Volume -5, No.1, and Feb. 2013. , Shreyash Des

[8] Yasir Rahmatallah, Member IEEE, Seshadri Mohan, Member IEEE "Peak to Average Power Ratio Reduction in OFDM Systems: A Survey and Texonomy" IEEE Communication Surveys and Tutorials, 2013.

[9] Kamal Singh, Abhinav Dogra, and Gopal Singh Naryal "A New Phase Sequence PAPR Reduction Technique Based on SLM and PTS Techniques" International Journal of IT, Engineering and Applied Science Research (IJIEASR), ISSN: 2319-4413, Volume -1, No.3, and December 2012.

[10] L.Yang, K.K.Soo, S.Q.Li and Y.M.Siu "PAPR Reduction using Low Complexity PTS to Construct of OFDM Signals without Side Information", IEEE Transactions on Broadcasting, Volume -57, No.2, and June 2011

[11] M. Sifuzzaman, M.R.Islam and M.Z.Ali "Application of Wavelet Transform and its Advantage Compare to Fourier Transform", Journal of Physical Science, ISSN: 0972-8791, Volume-13, October 2009.

[12] S.H. Muller and J.B. Huber "A comparison of Peak Power Reduction Schemes for OFDM". In Globecom 97, Phoenix, USA, 1997. 